\title{
Absence of Triplets in Single-Photon Double Ionization of Methanol
}

\author{
Krishnendu Gope ${ }^{\S}$ Ester Livshits, ${ }^{\S}$ Dror M. Bittner, Roi Baer, and Daniel Strasser*
}

Cite This: J. Phys. Chem. Lett. 2020, 11, 8108-8113

Read Online

ABSTRACT: Despite the abundance of data concerning single-photon double ionization of methanol, the spin state of the emitted electron pair has never been determined. Here we present the first evidence that identifies the emitted electron pair spin as overwhelmingly singlet when the dication forms in low-energy configurations. The experimental data show that while the yield of the $\mathrm{CH}_{2} \mathrm{O}^{+}+\mathrm{H}_{3}{ }^{+}$Coulomb explosion channel is abundant, the metastable methanol dication is largely absent. According to high-level ab initio simulations, these facts indicate that photoionization promptly forms singlet dication states, where they quickly decompose through various channels, with significant $\mathrm{H}_{3}{ }^{+}$yields on the low-lying states. In contrast, if we assume that the initial dication is formed in one of the low-lying triplet states, the $\mathrm{ab}$ initio simulations exhibit a metastable dication, contradicting the experimental findings. Comparing the average simulated branching ratios with the experimental data suggests a $>3$ order of magnitude enhancement of the singlet:triplet ratio compared with their respective 1:3 multiplicities.

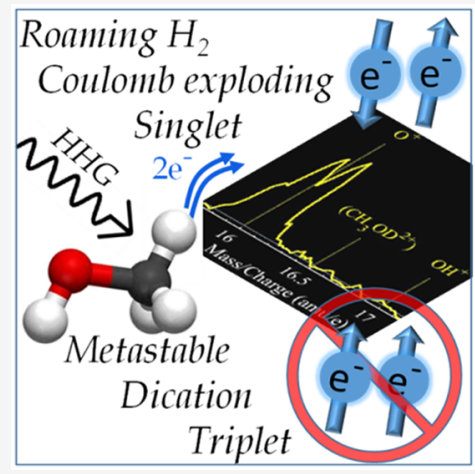

$\mathrm{P}$ hotoionization is one of the basic types of light-matter interaction. The most intensely studied and implemented scenario is the single-photon-induced emission of an electron at a rate that is linear with light intensity. ${ }^{1-8}$ In contrast, there are several types of photoemission processes in which one photon induces the emission of two (or more) electrons. These are classified by the excitation mechanism, for example, the Auger decay ${ }^{9-13}$ or the interatomic Coulombic decay ${ }^{14-18}$ following ionization of a core electron. A less well-understood category of single-photon double ionization (SPDI) is the concerted removal of two valence electrons by a single EUV photon that relies on electron correlations. ${ }^{19-22}$ In molecular systems, intramolecular charge migration on the attosecond time scale initiated by SPDI affects the molecular dynamics and Coulomb explosion (CE) that follow on the femtosecond time scale. ${ }^{23-33}$ SPDI is closely related to the single-photoninduced multiexciton generation (MEG) phenomenon appearing in solids and quantum-confined nanostructures and affecting the efficiency of solar cell devices. ${ }^{34,35}$

The determination of the spin state of the ejected electrons in SPDI is central to understanding the process, but its determination is a challenge. Some SPDI studies have exploited symmetry for determining the total spin state of the emitted electrons. For example, in isolated centrosymmetric systems, the ejected electron pairs in triplet-ungerade or singlet-gerade wave functions exhibit a node at the so-called "Wannier point" and are therefore less likely to be produced by SPDI. ${ }^{36-40}$ This propensity rule was supported by observation of the electronic state of the remaining dication. Gaire et al. compared the near-threshold double photoionization of two centrosymmetric systems, ${ }^{41}$ acetylene and ethylene, using the measured photoelectron energies and photo-ion masses to

determine the final state of the dication. For the acetylene dication, a highly populated metastable ${ }^{3} \sum_{g}{ }^{-}$ground state was found, while for ethylene, the metastable ${ }^{1} \mathrm{~A}_{\mathrm{g}}$ ground state was barely populated. These results were in accordance with the propensity rule. $^{41}$

However, in molecules that are not centrosymmetric, little is known about the total spin state formed in the SPDI processes. For example, SPDI measurements in methanol, ${ }^{42}$ among other noncentrosymmetric systems, ${ }^{21,42-44}$ were assigned to both singlet and triplet states of the emitted electron pair. This approach was adopted also by our previously published calculations of the methanol dication singlet and triplet potentials at fixed geometries as a function of the $\mathrm{C}-\mathrm{O}$ stretch, which suggested similar CT barriers for both singlet and triplet states. ${ }^{45}$ In the absence of inversion symmetry and very similar energetics in the Franck-Condon (FC) region, a 1:3 singlet:triplet ratio was tentatively suggested according to their respective multiplicities. ${ }^{45}$

In this paper, we focus on the spin state of the electron pair emitted in SPDI of methanol and find that previous assumptions about the spin propensities are not valid. In view of the absence of symmetry and the difficulties in direct measurements, we rely on non-adiabatic ab initio molecular dynamics (NA-AIMD) simulations as the primary tool for analyzing the experimental results. We have shown recently

Received: August 10, 2020

Accepted: September 8, 2020

Published: September 8, 2020 
that NA-AIMD simulations of the methanol singlet dication manifold successfully reproduce the experimental CE branching ratios, kinetic energy release (KER), and three-body momentum correlations, initiated by an ultrafast EUV pulse. ${ }^{27}$ High-lying excited states of the dication formed in SPDI were found to be responsible for the $\mathrm{C}-\mathrm{O}$ bond cleavage, either by rapid non-adiabatic electron transfer or by ultrafast proton migration from the doubly ionized methyl part to the $\mathrm{OH}$, while in the low-lying states, the initial charge localization on the methyl part and a charge-transfer (CT) barrier prevent the $\mathrm{C}-\mathrm{O}$ bond cleavage on the low-lying states, resulting in more complex dynamics, including the formation of $\mathrm{H}_{3}{ }^{+}$via a roaming $\mathrm{H}_{2}$ mechanism. The movie provided in the Supporting Information compares two typical singlet and triplet ground state trajectories, both initiated with the same phase-space configuration. While the triplet remains stable for $>1.5$ ps of the NA-AIMD simulation, the longest-lived singlet trajectory dissociates after less than $\sim 400 \mathrm{fs}$ due to the ultrafast roaming $\mathrm{H}_{2}$ dynamics. ${ }^{28}$ This in agreement with ultrafast $\mathrm{H}_{3}{ }^{+}$ formation determined by time-resolved experimental studies. $^{28,46}$ In contrast, all of the 100 NA-AIMD trajectory simulations performed on the triplet ground state were found to resist $\mathrm{CE}$.

In Figure 1, we show the relative energetics of the singlet and triplet ground state potentials at different molecular

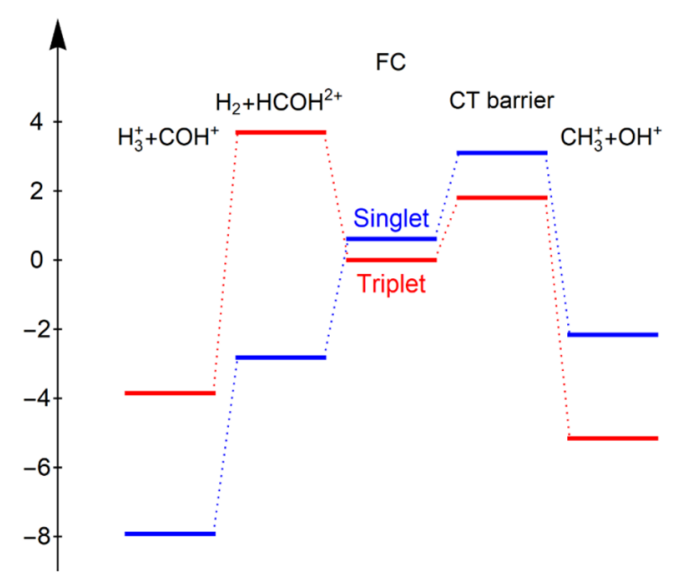

Figure 1. Methanol dication system energitics compared to the relaxed $\mathrm{H}_{3}^{+}+\mathrm{COH}^{+}$and $\mathrm{CH}_{3}^{+}+\mathrm{OH}^{+}$dissociation limits using B3LYP/d-aug-cc-pVTZ calculations of the separately optimized molecular geometries of the dissociation products. Singlet and triplet states are shown by blue solid and red dashed lines, respectively. FC indicates the Frack-Condon geometry of the neutral, CT the barrier charge transfer, and the $\mathrm{H}_{2}+\mathrm{HCOH}^{2+}$ limit the roaming neutral $\mathrm{H}_{2}$ state.

geometries. At the Franck-Condon (FC) geometry, the triplet manifold's ground state lies slightly below that of the singlet. Both triplet and singlet states exhibit similar $\sim 3 \mathrm{eV}$ CT barriers, effectively preventing the $\mathrm{C}-\mathrm{O}$ bond's cleavage from forming the $\mathrm{CH}_{3}{ }^{+}+\mathrm{OH}^{+}$products. On the singlet ground state, the formation of $\mathrm{H}_{3}^{+}+\mathrm{COH}^{+}$is energetically favorable and proceeds efficiently via the roaming $\mathrm{H}_{2}$ mechanism. ${ }^{46-49}$ Here, a neutral $\mathrm{H}_{2}$ separates from a $\mathrm{HCOH}^{2+}$ dication decays by competing channels of proton capture, forming $\mathrm{H}_{3}{ }^{+}$, and long-range electron transfer ("inverse harpooning"), forming $\mathrm{H}_{2}{ }^{+27,28}$ When considering the triplet state energetics along a possible roaming $\mathrm{H}_{2}$ pathway, we need to remember that $\mathrm{H}_{2}$ is stable only in its singlet state, hence requiring a triplet
$\mathrm{HCOH}^{2+}$ As shown in Figure 1, the optimized $\mathrm{HCOH}^{2+}$ triplet state lies over $5.9 \mathrm{eV}$ above that of the singlet, posing an $\sim 3.7 \mathrm{eV}$ barrier that prevents triplet methanol dications formed in the FC geometry from dissociating via the roaming $\mathrm{H}_{2}$ mechanism. In comparison, singlet $\mathrm{HCOH}^{2+}+\mathrm{H}_{2}$ lies $\sim 2.8$ $\mathrm{eV}$ below the singlet $\mathrm{FC}$ geometry, resulting in energetically favorable roaming $\mathrm{H}_{2}$ dynamics.

As predicted by Luzon et al., ${ }^{45}$ similarly to the high-lying singlet states, high-lying triplets can fragment via cleavage of the $\mathrm{C}-\mathrm{O}$ bond, eliminating metastable trajectories. However, although $\mathrm{H}_{2}{ }^{+}$is observed to form on excited states of the triplet manifold (via nonroaming dynamics), $\mathrm{H}_{3}{ }^{+}$was not formed in any of the 700 trajectories simulated on the ground state and the first six excited states of the triplet manifold. In Table 1, we summarize the probabilities for formation of $\mathrm{H}_{3}{ }^{+}$and methanol dication $\mathrm{CH}_{3} \mathrm{OH}^{2+}$ in its singlet and triplet states.

Table 1. Comparison of Experimentally Measured and State-Resolved Theoretically Simulated Branching Ratios for the $\mathrm{H}_{3}{ }^{+}+\mathrm{COH}^{+}$Coulomb Explosion and the Metastable $\mathrm{CH}_{3} \mathrm{OH}^{2+}$ Dication

\begin{tabular}{|c|c|c|c|}
\hline & & $\begin{array}{c}\mathrm{H}_{3}^{+}+ \\
\mathrm{COH}^{+}(\%)\end{array}$ & $\begin{array}{c}\mathrm{CH}_{3} \mathrm{OH}^{2+} \\
(\%)\end{array}$ \\
\hline \multirow[t]{2}{*}{ theory } & $\begin{array}{l}\text { singlet (GS, first, second, third, } \\
\text { fourth-sixth) }\end{array}$ & $\begin{array}{l}34,21,7 \\
\quad 7,0\end{array}$ & - \\
\hline & $\begin{array}{l}\text { triplet (GS, first, second, third, } \\
\text { fourth-sixth) }\end{array}$ & - & $\begin{array}{l}99,41,16 \\
\quad 6,0\end{array}$ \\
\hline \multirow[t]{2}{*}{ experiment } & undeuterated & $6 \pm 0.5$ & $<1$ \\
\hline & deuterrated $^{a}$ & $8 \pm 0.5$ & $<0.035$ \\
\hline
\end{tabular}

The exclusive production of $\mathrm{H}_{3}^{+}$on singlet states and metastable dications on triplet states opens an opportunity for experimental determination of the singlet:triplet ratio in the SPDI of $\mathrm{CH}_{3} \mathrm{OH}$. However, the $32 / 2$ mass over charge ratio of the methanol dication is accidentally equal to that of the $\mathrm{O}^{+}$ ion. Nevertheless, three-dimensional (3D) fragment imaging allows us to detect the momentum of each ion as well as the mass/charge ratio of the photoionization product based on the time-of-flight measurement. Figure 2a shows the momentum distribution of the parent $\mathrm{CH}_{3} \mathrm{OH}^{+}$ion measured in the $X-Y$ plane of the microchannel plate (MCP) detector. The red ellipse indicates ions originating from the molecular beam, which are moving in the $Y$ direction of the lab frame. The dashed magenta circle indicates the momenta of $\mathrm{CH}_{3} \mathrm{OH}^{+}$ions formed from the residual gas, showing a broader and isotropically oriented distribution compared to that of the skimmed molecular beam. In contrast, Figure $2 \mathrm{~b}$ shows the momentum distribution measured for a mass/charge ratio of $32 / 2$ corresponding to either metastable $\mathrm{CH}_{3} \mathrm{OH}^{2+}$ dications or $\mathrm{O}^{+}$fragments. Clearly, the signal is dominated by the $\mathrm{O}^{+}$ product of dissociative ionization, whose recoil results in the observed broad momentum distribution.

To disentangle the metastable $\mathrm{CH}_{3} \mathrm{OH}^{2+}$ signal, we take advantage of the negligible recoil of the parent ion due to electron ejection. Metastable $\mathrm{CH}_{3} \mathrm{OH}^{2+}$ ions originating from the molecular beam are expected to exhibit a momentum distribution similar to that of the $\mathrm{CH}_{3} \mathrm{OH}^{+}$ions, shown by the red ellipse in Figure 2a. Furthermore, we can subtract an average background of low-recoil fragments, estimated from the regions indicated by the dotted black ellipses in panels a and $b$ of Figure 2. The blue line in Figure $2 c$ shows the mass/ 

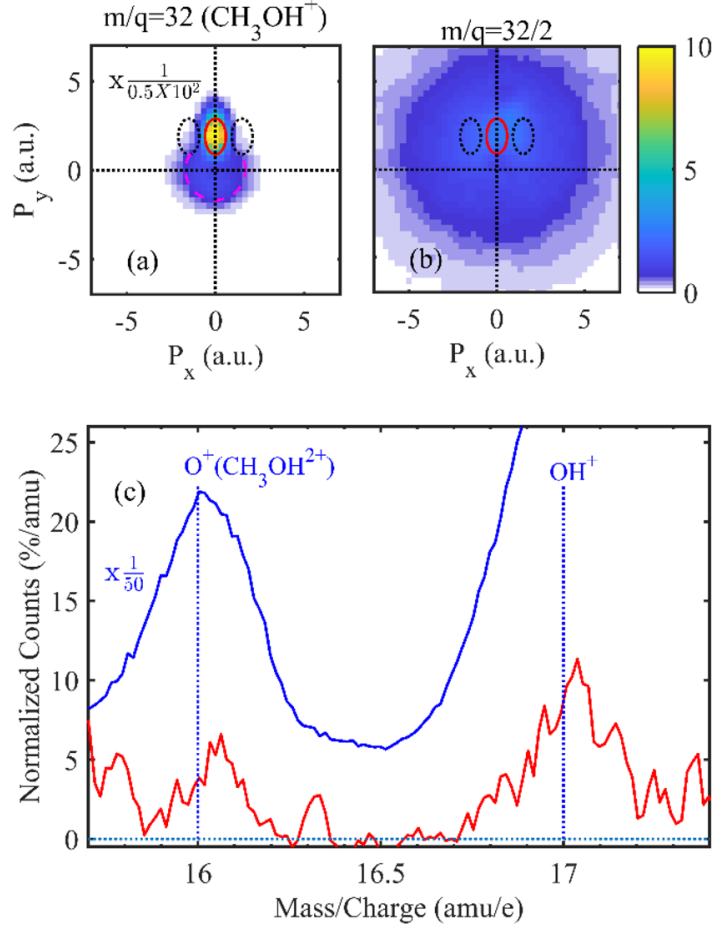

Figure 2. Momentum images of (a) $m / q=32$ (undissociated $\mathrm{CH}_{3} \mathrm{OH}^{+}$) and (b) $m / q=32 / 2$. Expected momentum regions of the molecular beam and residual gas are shown by solid red and dashed magenta regions, respectively. (c) Total and background-subtracted product-normalized counts from $\mathrm{CH}_{3} \mathrm{OH}^{2+}$ initiated by single-photon EUV with respect to the total yield of CE channels are shown by blue and red lines, respectively.

charge spectrum of the total ion yield $\times 1 / 50$, where the red line shows the background-subtracted spectrum of ions formed with no recoil from the molecular beam. Both spectra are normalized to the total yield of CE channels that include $\mathrm{CO}$ bond cleavage, $\mathrm{H}_{n}{ }^{+}$formation, and different three-body $\mathrm{CE}$ channels. ${ }^{27}$ The background-subtracted mass spectrum exhibits residual contributions from low-KER $\mathrm{OH}^{+}$and $\mathrm{O}^{+}$(or $\mathrm{CH}_{3} \mathrm{OH}^{2+}$ ), allowing determination of an upper limit of $<1 \%$ probability for the formation of a metastable methanol dication in single-photon double ionization. In comparison, $\mathrm{H}_{3}{ }^{+}$ formation is observed at $\sim 6 \%$ of all CE events, in agreement with the results of Luzon et al. ${ }^{27}$

To remove the residual uncertainty due to low $\mathrm{KER} \mathrm{\textrm {O } ^ { + }}$ yields, we performed the same study using deuterated $\mathrm{CH}_{3} \mathrm{OD}$ methanol. Panels a and b of Figure 3 show the momentum distributions corresponding to mass over charge values of 33 and $33 / 2$, which can be distinguished from singly ionized products. Note the 50 -fold lower yield color scale values for a $33 / 2$ mass/charge ratio compared with Figure $2 b$, both normalized to the respective singly ionized parent cation yields. Due to the improved signal-to-noise ratio and statistics of the $\mathrm{CH}_{3} \mathrm{OD}$ measurement, the low $\mathrm{KER} \mathrm{\textrm {O } ^ { + }}$ peak is clearly visible in the mass spectrum shown in Figure 3c, showing a distinguishable splitting between $\mathrm{O}^{+}$fragments ejected toward or away from the MCP detector. As the deuteration is on the hydroxyl, $\mathrm{OH}^{+}$formation is strongly quenched, indicating the absence of complex carboxyl and hydroxyl swapping dynamics. ${ }^{50}$ The statistical error of the background-subtracted signal at a mass to charge ratio of $33 / 2$ allows an improved $0.035 \%$ upper limit for the metastable dication formation
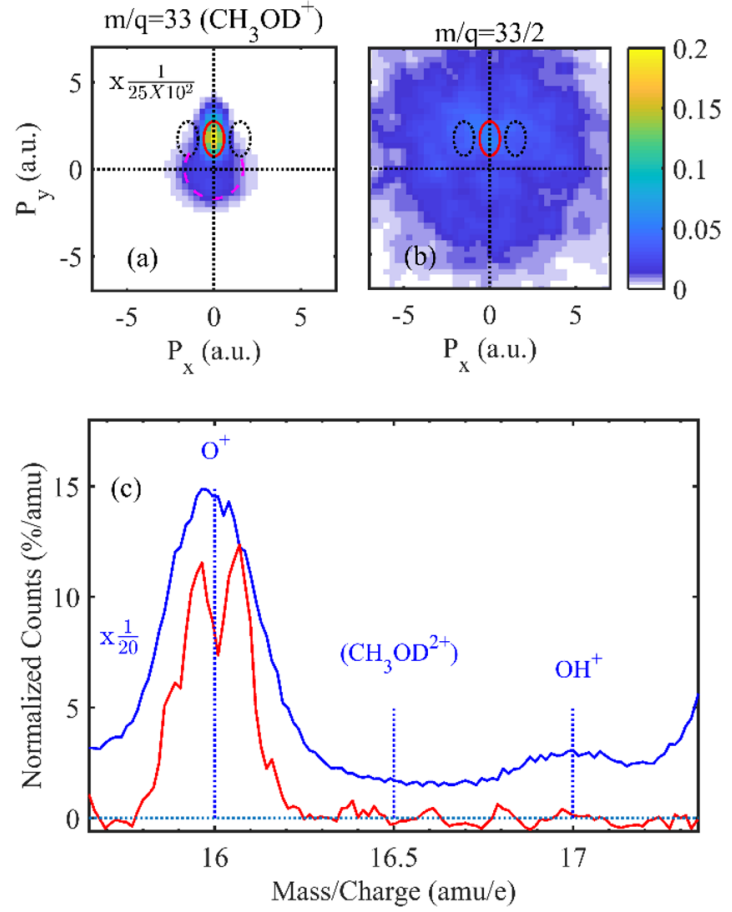

Figure 3. Momentum images of (a) $m / q=33$ (undissociated $\mathrm{CH}_{3} \mathrm{OD}^{+}$) and (b) $m / q=33 / 2$. Expected momentum regions of the molecular beam and residual gas are shown by solid red and dashed magenta regions, respectively. (c) Total and background-subtracted product-normalized counts from $\mathrm{CH}_{3} \mathrm{OD}^{2+}$ initiated by single-photon EUV with respect to the total yield of CE channels are shown by blue and red lines, respectively.

probability with respect to total CE channels yield following single-photon double ionization. The combined yields of $\mathrm{H}_{3}{ }^{+}$ and $\mathrm{H}_{2} \mathrm{D}^{+}$in the deuterated system amount to $\sim 8 \%$, slightly higher than the value of the measured $6 \% \mathrm{H}_{3}^{+}$formation branching ratio in the undeuterated system. As the deuterium is on the hydroxyl part, we assume a negligible isotope effect on the singlet:triplet ratio, roaming $\mathrm{H}_{2}$ or proton capture from the methyl part. Nevertheless, deuteration may affect the probability of the roaming $\mathrm{H}_{2}$ to capture the $\mathrm{H} / \mathrm{D}$ of the hydroxyl.

Assuming an equal population of the simulated states listed in Table 1 , the average $\mathrm{H}_{3}{ }^{+}$formation probability is expected to be $\sim 10 \%$ on the singlet states, in rough agreement with the experimental branching ratio. $\mathrm{No}_{3}{ }^{+}$formation is expected on the triplet manifold. In contrast, metastable dications are expected to form with an average $\sim 20 \%$ probability on an equally populated triplet manifold. Thus, assuming a single$t$ triplet ratio of $1: 3$ would result in six metastable cations for each $\mathrm{H}_{3}{ }^{+}+\mathrm{COH}^{+} \mathrm{CE}$ event. In contrast, the experimental data of the deuterated system show fewer than one metastable cation for more than $\sim 200$ triatomic hydrogen ions. This indicates a $>3$ order of magnitude enhancement relative to the tentatively expected singlet:triplet ratio according to their 1:3 multiplicities.

This surprising selectivity for single-photon double ionization of a noncentrosymmetric system suggests the possibility of new selection rules for single-photon double ionization. Nevertheless, it is important to note that while the NAAIMD simulations exhibit a stable dication up to $1.5 \mathrm{ps}$, the experimental setup cannot distinguish between instantaneous dissociation and delayed dissociation on a nanosecond time 
scale due to the finite acceleration time. Thus, a particularly efficient intersystem crossing, which is beyond the scope of the theoretical work presented here, could in principle quench the metastable dication. However, in the case of such significant intersystem crossing, the branching ratios, KER, and threebody momentum correlation distributions can be expected to reflect the landscape of the intersystem coupling and be quite different from the simulated singlet manifold dynamics that successfully reproduce the experimental data when initiated from the FC region. ${ }^{27}$

The presented singlet:triplet ratio enhancement in the single-photon double ionization of methanol provides insight into the electron-electron correlations induced in the SPDI process in the absence of inversion symmetry. The EUV photon energies produced by the HHG source lie high above the SPDI threshold, reaching $\sim 100 \mathrm{eV}$ photons. ${ }^{45}$ Thus, these findings are not limited to a near threshold region or a specific narrow window of photon energies. Future theoretical work is needed to investigate SPDI electron-electron correlations and describe the relative contribution of different excited states and to perform estimation of the possible intersystem crossing effect on the dication dynamics. The generality of the observed spin state selectivity can be further tested as suppression of roaming $\mathrm{H}_{2}$ dynamics on the triplet dication can be expected to play an important role also for other organic molecules that often exhibit formation of $\mathrm{H}_{3}{ }^{+}$via roaming $\mathrm{H}_{2}$ dynamics. ${ }^{46,49}$ Furthermore, double ionization by a strong-field laser, as opposed to ionization by a single EUV photon, may exhibit formation of metastable methanol dications and reveal different correlations between the ejected electrons.

\section{EXPERIMENTAL AND COMPUTATIONAL METHODS}

The experimental setup for single-photon Coulomb explosion imaging and theoretical NA-AIMD simulations have been described previously. ${ }^{27,32,45}$ Briefly, broad bandwidth ultrafast EUV pulses are produced by high-order harmonic generation (HHG) in a semi-infinite neon gas cell. ${ }^{51,52}$ The resulting EUV pulses are spatially filtered from the higher divergence near $\mathrm{IR}^{53}$ and cross a skimmed effusive beam of commercially available $\mathrm{CH}_{3} \mathrm{OH}$ or $\mathrm{CH}_{3} \mathrm{OD}$ samples. The cationic products are accelerated, and their coincidence 3D momenta are imaged on a time and position sensitive detector. ${ }^{45,54}$ True CE events are disentangled from an overwhelming dissociative ionization background based on the total momentum conservation of two coincident hits on the detector. ${ }^{45}$

Theoretical NA-AIMD simulations were performed using CASPT2 potentials. ${ }^{55-60}$ Non-adiabatic dynamics were approximated using surface-hopping molecular dynamics trajectories, ${ }^{55}$ generated at the XMS-CASPT2/(8e,8o $) /$ augcc-pVDZ level using the BAGEL electronic structure package. $^{56}$ A more detailed description of the NA-AIMD is provided in our previous work concerning the CE dynamics on the singlet manifold of methanol dication states. ${ }^{27}$ To determine the relative probability of different double-ionization product channels, for each dication state, we initiate 100 trajectories from different initial phase-space configurations. These were sampled from neutral ground state AIMD simulations at $300 \mathrm{~K}$, performed on the CASSCF level using the MOLCAS package. ${ }^{58}$

\section{ASSOCIATED CONTENT}

\section{Supporting Information}

The Supporting Information is available free of charge at https://pubs.acs.org/doi/10.1021/acs.jpclett.0c02445.

Movie comparing two typical singlet and triplet ground state trajectories, both initiated with the same phasespace configuration (MP4)

\section{AUTHOR INFORMATION}

\section{Corresponding Author}

Daniel Strasser - Institute of Chemistry, The Hebrew University of Jerusalem, Jerusalem 91904, Israel; (1) orcid.org/00000002-9000-3816; Email: strasser@huji.ac.il

\section{Authors}

Krishnendu Gope - Institute of Chemistry, The Hebrew University of Jerusalem, Jerusalem 91904, Israel

Ester Livshits - Fritz Haber Research Center for Molecular Dynamics and Institute of Chemistry, The Hebrew University of Jerusalem, Jerusalem 91904, Israel

Dror M. Bittner - Institute of Chemistry, The Hebrew University of Jerusalem, Jerusalem 91904, Israel

Roi Baer - Fritz Haber Research Center for Molecular Dynamics and Institute of Chemistry, The Hebrew University of Jerusalem,

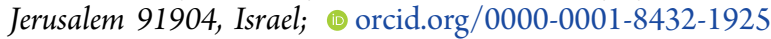

Complete contact information is available at:

https://pubs.acs.org/10.1021/acs.jpclett.0c02445

\section{Author Contributions}

${ }^{\S}$ K.G. and E.L. contributed equally to this work.

Notes

The authors declare no competing financial interest.

\section{ACKNOWLEDGMENTS}

The authors acknowledge the European Research Council (Grant 306783) and ISF Grant 1369/17 as well as equipment from the Wolfson Foundation. R.B. acknowledges support from Israel Science Foundation Grant ISF-800/19.

\section{REFERENCES}

(1) Ng, C.-Y. Vacuum Ultraviolet Spectroscopy And Chemistry By Photoionization And Photoelectron Method. Annu. Rev. Phys. Chem. 2002, 53 (1), 101-140.

(2) Klünder, K.; Dahlström, J. M.; Gisselbrecht, M.; Fordell, T.; Swoboda, M.; Guénot, D.; Johnsson, P.; Caillat, J.; Mauritsson, J.; Maquet, A.; et al. Probing Single-Photon Ionization on the Attosecond Time Scale. Phys. Rev. Lett. 2011, 106 (14), 143002.

(3) Qi, F. Combustion Chemistry Probed by Synchrotron VUV Photoionization Mass Spectrometry. Proc. Combust. Inst. 2013, 34 (1), $33-63$.

(4) Berkowitz, J.; Ellison, G. B.; Gutman, D. Three Methods to Measure RH Bond Energies. J. Phys. Chem. 1994, 98 (11), 27442765.

(5) Downie, P.; Powis, I. Molecule-Frame Photoelectron Angular Distributions from Oriented CF3I Molecules. Phys. Rev. Lett. 1999, 82 (14), 2864-2867.

(6) Garcia, G. A.; Nahon, L.; Daly, S.; Powis, I. Vibrationally Induced Inversion of Photoelectron Forward-Backward Asymmetry in Chiral Molecule Photoionization by Circularly Polarized Light. Nat. Commun. 2013, 4 (1), 2132.

(7) Strasser, D.; Goulay, F.; Leone, S. R. Transient Photoelectron Spectroscopy of the Dissociative $\mathrm{Br}_{2}\left({ }^{1} \Pi_{\mathrm{u}}\right)$ State. J. Chem. Phys. 2007, 127 (18), 184305. 
(8) Ossiander, M.; Riemensberger, J.; Neppl, S.; Mittermair, M.; Schäffer, M.; Duensing, A.; Wagner, M. S.; Heider, R.; Wurzer, M.; Gerl, M.; et al. Absolute Timing of the Photoelectric Effect. Nature 2018, 561 (7723), 374-377.

(9) Ågren, H.; Cesar, A.; Liegener, C.-M. Theory of Molecular Auger Spectra. Adv. Quantum Chem. 1992, 23, 1-82.

(10) Rye, R. R.; Madey, T. E.; Houston, J. E.; Holloway, P. H. Chemical-state Effects in Auger Electron Spectroscopy. J. Chem. Phys. 1978, 69 (4), 1504-1512.

(11) Thompson, M.; Hewitt, P. A.; Wooliscroft, D. S. Analytical Aspects of Auger Electron Spectrometry of Gases. Anal. Chem. 1976, 48 (9), 1336-1340.

(12) Schins, J. M.; Breger, P.; Agostini, P.; Constantinescu, R. C.; Muller, H. G.; Grillon, G.; Antonetti, A.; Mysyrowicz, A. Observation of Laser-Assisted Auger Decay in Argon. Phys. Rev. Lett. 1994, 73 (16), 2180-2183.

(13) Moddeman, W. E.; Carlson, T. A.; Krause, M. O.; Pullen, B. P.; Bull, W. E.; Schweitzer, G. K. Determination of the K-LL Auger Spectra of N 2, O 2, CO, NO, H 2 O, and CO 2. J. Chem. Phys. 1971, 55 (5), 2317-2336.

(14) Marburger, S.; Kugeler, O.; Hergenhahn, U.; Möller, T. Experimental Evidence for Interatomic Coulombic Decay in $\mathrm{Ne}$ Clusters. Phys. Rev. Lett. 2003, 90 (20), 203401.

(15) Averbukh, V.; Müller, I. B.; Cederbaum, L. S. Mechanism of Interatomic Coulombic Decay in Clusters. Phys. Rev. Lett. 2004, 93 (26), 263002.

(16) Hempelmann, A.; Piancastelli, M. N.; Heiser, F.; Gessner, O.; Rüdel, A.; Becker, U. Resonant Photofragmentation of Methanol at the Carbon and Oxygen K-Edge by High-Resolution Ion-Yield Spectroscopy. J. Phys. B: At., Mol. Opt. Phys. 1999, 32 (11), 26772689.

(17) Schnorr, K.; Senftleben, A.; Kurka, M.; Rudenko, A.; Foucar, L.; Schmid, G.; Broska, A.; Pfeifer, T.; Meyer, K.; Anielski, D.; et al. Time-Resolved Measurement of Interatomic Coulombic Decay in $\mathrm{Ne}_{2}$. Phys. Rev. Lett. 2013, 111 (9), 093402.

(18) Cederbaum, L. S.; Zobeley, J.; Tarantelli, F. Giant Intermolecular Decay and Fragmentation of Clusters. Phys. Rev. Lett. 1997, 79 (24), 4778-4781.

(19) Weber, T.; Czasch, A. O.; Jagutzki, O.; Müller, A. K.; Mergel, V.; Kheifets, A.; Rotenberg, E.; Meigs, G.; Prior, M. H.; Daveau, S.; et al. Complete Photo-Fragmentation of the Deuterium Molecule. Nature 2004, 431 (7007), 437-440.

(20) Ueda, K.; Eland, J. H. D. Molecular Photodissociation Studied by VUV and Soft X-Ray Radiation. J. Phys. B: At., Mol. Opt. Phys. 2005, 38 (9), S839-S859.

(21) Eland, J. H. D. Complete Double Photoionisation Spectra of Small Molecules from TOF-PEPECO Measurements. Chem. Phys. 2003, 294 (2), 171-186.

(22) Lago, A. F.; Santos, A. C. F.; de Souza, G. G. B. Mass Spectrometry Study of the Fragmentation of Valence and Core-Shell (Cl 2p) Excited $\mathrm{CHCl} 3$ and $\mathrm{CDCl} 3$ Molecules. J. Chem. Phys. 2004, 120 (20), 9547-9555.

(23) Ayuso, D.; Palacios, A.; Decleva, P.; Martín, F. Ultrafast Charge Dynamics in Glycine Induced by Attosecond Pulses. Phys. Chem. Chem. Phys. 2017, 19 (30), 19767-19776.

(24) Lara-Astiaso, M.; Galli, M.; Trabattoni, A.; Palacios, A.; Ayuso, D.; Frassetto, F.; Poletto, L.; De Camillis, S.; Greenwood, J.; Decleva, P.; et al. Attosecond Pump-Probe Spectroscopy of Charge Dynamics in Tryptophan. J. Phys. Chem. Lett. 2018, 9 (16), 4570-4577.

(25) Sansone, G.; Kelkensberg, F.; Pérez-Torres, J. F.; Morales, F.; Kling, M. F.; Siu, W.; Ghafur, O.; Johnsson, P.; Swoboda, M.; Benedetti, E.; et al. Electron Localization Following Attosecond Molecular Photoionization. Nature 2010, 465 (7299), 763-766.

(26) Remacle, F.; Levine, R. D. Attosecond Pumping of Nonstationary Electronic States of $\mathrm{LiH}$ : Charge Shake-up and Electron Density Distortion. Phys. Rev. A: At., Mol., Opt. Phys. 2011, 83 (1), 013411.
(27) Luzon, I.; Livshits, E.; Gope, K.; Baer, R.; Strasser, D. Making Sense of Coulomb Explosion Imaging. J. Phys. Chem. Lett. 2019, 10 (6), 1361-1367.

(28) Livshits, E.; Luzon, I.; Gope, K.; Baer, R.; Strasser, D. TimeResolving the Ultrafast $\mathrm{H} 2$ Roaming Chemistry and $\mathrm{H} 3+$ Formation Using Extreme-Ultraviolet Pulses. Commun. Chem. 2020, 3 (1), 49.

(29) Jiang, Y. H.; Rudenko, A.; Herrwerth, O.; Foucar, L.; Kurka, M.; Kühnel, K. U.; Lezius, M.; Kling, M. F.; van Tilborg, J.; Belkacem, A.; et al. Ultrafast Extreme Ultraviolet Induced Isomerization of Acetylene Cations. Phys. Rev. Lett. 2010, 105 (26), 263002.

(30) Lehmann, C. S.; Picón, A.; Bostedt, C.; Rudenko, A.; Marinelli, A.; Moonshiram, D.; Osipov, T.; Rolles, D.; Berrah, N.; Bomme, C.; et al. Ultrafast X-Ray-Induced Nuclear Dynamics in Diatomic Molecules Using Femtosecond x-Ray-Pump-x-Ray-Probe Spectroscopy. Phys. Rev. A: At., Mol., Opt. Phys. 2016, 94 (1), 013426.

(31) Ablikim, U.; Bomme, C.; Xiong, H.; Savelyev, E.; Obaid, R.; Kaderiya, B.; Augustin, S.; Schnorr, K.; Dumitriu, I.; Osipov, T.; et al. Identification of Absolute Geometries of Cis and Trans Molecular Isomers by Coulomb Explosion Imaging. Sci. Rep. 2016, 6 (1), 38202.

(32) Gope, K.; Luzon, I.; Strasser, D. N-NO \& NN-O Bond Cleavage Dynamics in Two- and Three-Body Coulomb Explosion of the $\mathrm{N}_{2} \mathrm{O}^{2+}$ Dication. Phys. Chem. Chem. Phys. 2019, 21, 1373013737.

(33) Reedy, D.; Williams, J. B.; Gaire, B.; Gatton, A.; Weller, M.; Menssen, A.; Bauer, T.; Henrichs, K.; Burzynski, P.; Berry, B.; et al. Dissociation Dynamics of the Water Dication Following One-Photon Double Ionization. II. Experiment. Phys. Rev. A: At., Mol., Opt. Phys. 2018, 98 (5), 053430.

(34) Gordi, M.; Moravvej-Farshi, M. K.; Ramezani, H. Effects of Electric Fields on Multiple Exciton Generation. ChemPhysChem 2018, 19 (20), 2782-2787.

(35) Schaller, R. D.; Klimov, V. I. High Efficiency Carrier Multiplication in PbSe Nanocrystals: Implications for Solar Energy Conversion. Phys. Rev. Lett. 2004, 92 (18), 186601.

(36) Wannier, G. H. The Threshold Law for Single Ionization of Atoms or Ions by Electrons. Phys. Rev. 1953, 90 (5), 817-825.

(37) Stauffer, A. D. The Nodal Structure of Two-Electron Wave Functions on the Wannier Ridge. Phys. Lett. A 1982, 91 (3), 114116

(38) Greene, C. H.; Rau, A. R. P. Double Escape of Two Electrons at Threshold: Dependence on L, S and $\pi$. Phys. Rev. Lett. 1982, 48 (8), 533-537.

(39) Lablanquie, P.; Eland, J. H. D.; Nenner, I.; Morin, P.; Delwiche, J.; Hubin-Franskin, M. J. Threshold Behavior in Single-Photon Double Ionization of Argon. Phys. Rev. Lett. 1987, 58 (10), 992-995.

(40) Eland, J. H. D.; Price, S. D.; Cheney, J. C.; Lablanquie, P.; Nenner, I.; Fournier, P. G. Towards a Spectroscopy of Doubly Charged Ions. Philos. Trans. R. Soc. London. Ser. A, Math. Phys. Sci. 1988, 324 (1578), 247-255.

(41) Gaire, B.; Lee, S. Y.; Haxton, D. J.; Pelz, P. M.; Bocharova, I.; Sturm, F. P.; Gehrken, N.; Honig, M.; Pitzer, M.; Metz, D.; et al. Photo-Double-Ionization of Ethylene and Acetylene near Threshold. Phys. Rev. A: At., Mol., Opt. Phys. 2014, 89 (1), 013403.

(42) Linusson, P.; Stenrup, M.; Larson, Å.; Andersson, E.; Heijkenskjöld, F.; Andersson, P.; Eland, J. H. D.; Karlsson, L.; Rubensson, J.-E.; Feifel, R. Double Photoionization of Alcohol Molecules. Phys. Rev. A: At., Mol., Opt. Phys. 2009, 80 (3), 032516.

(43) Kraus, P. M.; Schwarzer, M. C.; Schirmel, N.; Urbasch, G.;

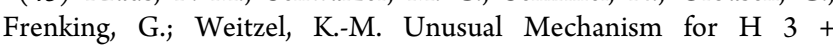
Formation from Ethane as Obtained by Femtosecond Laser Pulse Ionization and Quantum Chemical Calculations. J. Chem. Phys. 2011, 134 (11), 114302.

(44) Eland, J. H. D. The Origin of Primary H+3 Ions in Mass Spectra. Rapid Commun. Mass Spectrom. 1996, 10 (12), 1560-1562.

(45) Luzon, I.; Jagtap, K.; Livshits, E.; Lioubashevski, O.; Baer, R.; Strasser, D. Single-Photon Coulomb Explosion of Methanol Using Broad Bandwidth Ultrafast EUV Pulses. Phys. Chem. Chem. Phys. 2017, 19 (21), 13488-13495. 
(46) Ekanayake, N.; Nairat, M.; Kaderiya, B.; Feizollah, P.; Jochim, B.; Severt, T.; Berry, B.; Pandiri, K. R.; Carnes, K. D.; Pathak, S.; et al. Mechanisms and Time-Resolved Dynamics for Trihydrogen Cation $(\mathrm{H} 3+)$ Formation from Organic Molecules in Strong Laser Fields. Sci. Rep. 2017, 7 (1), 4703.

(47) Nakai, K.; Kato, T.; Kono, H.; Yamanouchi, K. Communication: Long-Lived Neutral H 2 in Hydrogen Migration within Methanol Dication. J. Chem. Phys. 2013, 139 (18), 181103.

(48) Mebel, A. M.; Bandrauk, A. D. Theoretical Study of Unimolecular Decomposition of Allene Cations. J. Chem. Phys. 2008, 129 (22), 224311.

(49) Ekanayake, N.; Severt, T.; Nairat, M.; Weingartz, N. P.; Farris, B. M.; Kaderiya, B.; Feizollah, P.; Jochim, B.; Ziaee, F.; Borne, K.; et al. $\mathrm{H}_{2}$ Roaming Chemistry and the Formation of $\mathrm{H}_{3}{ }^{+}$from Organic Molecules in Strong Laser Fields. Nat. Commun. 2018, 9 (1), 5186.

(50) Borkar, S.; Sztáray, B.; Bodi, A. Dissociative Photoionization Mechanism of Methanol Isotopologues $(\mathrm{CH} 3 \mathrm{OH}, \mathrm{CD} 3 \mathrm{OH}, \mathrm{CH} 3 \mathrm{OD}$ and CD3OD) by IPEPICO: Energetics, Statistical and Non-Statistical Kinetics and Isotope Effects. Phys. Chem. Chem. Phys. 2011, 13 (28), 13009.

(51) Sutherland, J.; Christensen, E.; Powers, N.; Rhynard, S.; Painter, J.; Peatross, J. High Harmonic Generation in a Semi-Infinite Gas Cell. Opt. Express 2004, 12 (19), 4430-4436.

(52) Brimhall, N.; Painter, J. C.; Powers, N.; Giraud, G.; Turner, M.; Ware, M.; Peatross, J. Measured Laser-Beam Evolution during HighOrder Harmonic Generation in a Semi-Infinite Gas Cell. Opt. Express 2007, 15 (4), 1684-1689.

(53) Salieres, P.; Ditmire, T.; Budil, K. S.; Perry, M. D.; L'Huillier, A. Spatial Profiles of High-Order Harmonics Generated by a Femtosecond Cr:LiSAF Laser. J. Phys. B: At., Mol. Opt. Phys. 1994, 27 (9), L217-L222.

(54) Kandhasamy, D. M.; Albeck, Y.; Jagtap, K.; Strasser, D. 3D Coincidence Imaging Disentangles Intense Field Double Detachment of $\mathrm{SF}^{6-}$. J. Phys. Chem. A 2015, 119 (29), 8076-8082.

(55) Park, J. W.; Shiozaki, T. On-the-Fly CASPT2 Surface-Hopping Dynamics. J. Chem. Theory Comput. 2017, 13 (8), 3676-3683.

(56) Shiozaki, T. BAGEL: Brilliantly Advanced General Electronicstructure Library. Wiley Interdiscip. Rev.: Comput. Mol. Sci. 2018, 8 (1), e1331.

(57) Barbatti, M.; Ruckenbauer, M.; Plasser, F.; Pittner, J.; Granucci, G.; Persico, M.; Lischka, H. Newton-X: A Surface-Hopping Program for Nonadiabatic Molecular Dynamics. Wiley Interdiscip. Rev. Comput. Mol. Sci. 2014, 4 (1), 26-33.

(58) Aquilante, F.; Autschbach, J.; Carlson, R. K.; Chibotaru, L. F.; Delcey, M. G.; De Vico, L.; Fdez. Galván, I.; Ferré, N.; Frutos, L. M.; Gagliardi, L.; et al. Molcas 8: New Capabilities for Multiconfigurational Quantum Chemical Calculations across the Periodic Table. J. Comput. Chem. 2016, 37 (5), 506-541.

(59) Tully, J. C. Molecular Dynamics with Electronic Transitions. J. Chem. Phys. 1990, 93 (2), 1061-1071.

(60) Vlaisavljevich, B.; Shiozaki, T. Nuclear Energy Gradients for Internally Contracted Complete Active Space Second-Order Perturbation Theory: Multistate Extensions. J. Chem. Theory Comput. 2016, 12 (8), 3781-3787. 